\title{
On Special Relativity with Cosmological Constant
}

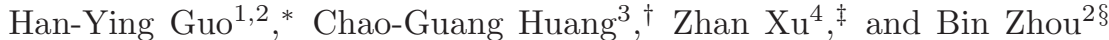 \\ ${ }^{1}$ CCAST (World Laboratory), P.O. Box 8730, Beijing 100080, China, \\ 2 Institute of Theoretical Physics, Chinese Academy of Sciences, P.O.Box 2735, Beijing 100080, China, \\ 3 Institute of High Energy Physics, Chinese Academy of Sciences, P.O. Box 918-4, Beijing 100039, China, and \\ 4 Physics Department, Tsinghua University, Beijing 100084, China.
}

(Dated: March 10, 2004)

\begin{abstract}
Based on the principle of relativity and the postulate of invariant speed and length, we propose the theory of special relativity with cosmological constant $\mathcal{S R}_{c, R}$ if the invariant length whose square is the inverse of the one-third cosmological constant of the universe. It is on the Beltrami-de Sitter spacetime $\mathcal{B}_{R}$ with de Sitter invariance. We define the observables of free particles and generalize famous Einstein's formula. We also define two kinds of simultaneity. The first is for local experiments and inertial motions. The second is for cosmological observations. Thus there is a relation between the relativity principle and the cosmological principle. We predict that the 3 - $\mathrm{d}$ cosmic space is then of positive spatial curvature of order cosmological constant. The relation between $\mathcal{S R}_{c, R}$ and the doubly special relativity is briefly disucssed.
\end{abstract}

PACS numbers: 03.30.+p, 98.80.Jk, 02.40.Dr, 02.40.Ky.

\section{INTRODUCTION}

Recent observations show that there should be a positive cosmological constant 1],2]. These lead to a lot of puzzles related to the de Sitter $(d S)$ space and may touch the foundation of physics [3].

In this letter, we generalize Einstein's special relativity $\mathcal{S} \mathcal{R}_{c}$ to the one with an additional invariant constant of length, $\mathcal{S R}_{c, R}$, based on the principle of relativity (PoR) and the postulate of invariant speed and length (PoI). With an identification of the length as $\sqrt{3 / \Lambda}$ where $\Lambda$ is the cosmological constant, it is the special relativity of the Beltrami-de Sitter spacetime $\mathcal{B}_{R}$ with de Sitter invariance [4], 5]. It is of course straightforward to set up its counterpart of anti-de Sitter(AdS) spacetime with an identification of the length as $\sqrt{-3 / \Lambda}$ ( For earlier works, see also 7], 8]).

It is related with some simple but important properties of $d S$ spacetime [6]-11]. In fact, among various metrics of $d S$ spacetime, there is an important one in which $d S$ spacetime is in analog with Minkowski spacetime. It is the $d S$ spacetime with Beltrami-like metric, called $B d S$ spacetime and denoted as $\mathcal{B}_{R}$. $B d S$ spacetime is precisely the Beltrami-like model [12] of a 4-hyperboloid $\mathcal{S}_{R}$ in 5-d Minkowski spacetime, i.e. $\mathcal{B}_{R} \backsim \mathcal{S}_{R}$. In $\mathcal{B}_{R}$ there exist a set of Beltrami coordinate systems, which covers $\mathcal{B}_{R}$ patch by patch, and in which test particles and light signals move along the timelike and null geodesics, respectively, with constant coordinate velocities. Therefore, they look like in free motion in a spacetime without gravity. Thus, the Beltrami coordinates and observers $\mathcal{O}_{B}$ at these systems should be regarded as of inertia.

\footnotetext{
*Electronic address: hyguo@itp.ac.cn

$\dagger$ Electronic address: huangcg@mail.ihep.ac.cn

¥Electronic address: zx-dmp@mail.tsinghua.edu.cn

$\S$ Electronic address: zhoub@itp.ac.cn
}

And the classical observables for these particles and signals can be well defined.

The key observation is that in a constant curvature spacetime there do exist such motions and observers of inertia. If we start with the 4-d Euclidean geometry and weaken the fifth axiom, then there should be 4-d Riemann, Euclid, and Lobachevski geometries at equal footing. Importantly, their geodesics are globally straight lines in certain coordinate systems that are just ones in analog with the coordinate systems in Beltrami model of Lobachevski plane [12], and under corresponding transformation groups the systems transform among themselves. Now changing the signature to -2 , these constant curvature spaces turn to be $d S$, Minkowski $(M)$, and $A d S$ spacetimes, respectively, and those straight lines are classified by timelike, null and spacelike straight worldlines. This may also be seen from viewpoint of projective geometry by an antipodal identified version of, say, $d S$ space time, $d S / Z_{2} \subset R P^{4}$, except the latter is neither orientable nor time orientable.

Thus, in analog with $\mathcal{S R}_{c}$, all of them should describe the inertial motions for free particles and light signals, respectively. And, Einstein's PoR should also be available for them, called the PoR with respect to $d S$, Poincaré, $A d S$ groups, respectively. In addition to the invariant speed, the speed of light, in $\mathcal{S} \mathcal{R}_{c}$ there is an invariant length $R$ in $d S$ and $A d S$, so the postulate of invariance of velocity of light in $\mathcal{S} \mathcal{R}_{c}$ should be replaced by the PoI. That is why based on these two principles, the special relativity with cosmological constant $\mathcal{S R}_{c, R}$ can be set up if the length being identified with $\sqrt{ \pm 3 / \Lambda}$.

The Beltrami coordinates make sense and concern with the measurements in laboratory at one patch, only if the simultaneity should be defined with respect to the Beltrami time coordinate. On the other hand, the simultaneity may also be defined by the proper time of a clock rest at the origin of Beltrami spatial coordinates. Thus, there are two kinds of simultaneity in $\mathcal{S} \mathcal{R}_{c, R}$ of $\mathcal{B}_{R}$. Consequently, if the simultaneity is transformed from the 
first to the second, the Beltrami metric is reduced to the Robertson-Walker(RW)-like metric in $\mathcal{B}_{R}$ with positive, rather than zero or negative, spatial curvature and its deviation from zero is in the order of cosmological constant $\Lambda$. Thus, the second simultaneity should be linked with the observation in the cosmological scale. This important property differs with either $\mathcal{S R}_{c}$ where two of them coincide, or usual cosmological models where $k$ is a free parameter. In fact, this is an important prediction. The tiny spatial closeness seems more or less to have been already confirmed by the CMB power spectrum data from WMAP [1] with error-control from SDSS 2] and should be further checked by its data in large scale.

Finally, we briefly mention that one may regard $\mathcal{S R}_{c, R}$ of $\mathcal{B}_{R}$ as a counterpart of the "doubly special relativity" (DSR) 13 in its momentum space as long as $R$ is taken as an observer-independent large-momentum scale.

This letter is organized as follows. In section II, we present two postulates and set up a framework for $\mathcal{S R}_{c, R}$ of $\mathcal{B}_{R}$. In section III we show explicitly the inertial motion of particles and light signals, define their observables and the simultaneity in inertial frame. In section IV, we define another kind of simultaneity with proper time and show that $\mathcal{B}_{R}$ can be reduced to RW-like metric with a slightly closed space. We end with a few remarks including the relation between the $\mathcal{S R}_{c, R}$ and the DSR.

\section{RELATIVITY WITH INVARIANT LENGTH}

We propose two fundamental principles as follows: The PoR requires there exist a set of inertial reference frames, in which the free particles and light signals move with uniform velocities along straight lines, the laws of nature without gravity are invariant under the transformations among them. The PoI requires there exist two invariant universal constants, one is with dimension of velocity, and another is of length.

It can be proved that the most general form of the transformations among inertial coordinate systems

$$
x^{\prime i}=f^{i}\left(x^{j}\right), \quad x^{0}=c t, \quad i, j=0, \cdots, 3,
$$

which transform a uniform-velocity, straight-line motion in $F$ to a motion of the same nature in $F^{\prime}$ are that the four functions $f^{i}$ are ratios of linear functions, all with the same denominators, where $c$ is the invariant speed 14.

As in $\mathcal{S} \mathcal{R}_{c}$, the PoR implicates that if there exists a metric on inertial frame at spacetime, it is of signature \pm 2 and invariant under some of these transformations that form a group with ten parameters including space-time "translations" (4), boosts (3) and space rotations (3), respectively. Thus, it can be proved that the necessary and sufficient condition for 4-d spacetimes with invariant metric of signature \pm 2 under ten-parameter transformation group is that they are $d S / M / A d S$ of positive, zero, or negative constant curvature, with the invariant group $S O(1,4)$, ISO $(1,3)$ or $S O(2,3)$, respectively. The PoI requires that there is a room for the constant $c$ as in (1) and the invariant length should be the curvature radius of $d S / A d S$, respectively.

We now consider the case of $d S$ spacetime, which can then be regarded as a 4-d hyperboloid $\mathcal{S}_{R}$ embedded in a 5-d Minkowski spacetime with $\eta_{A B}=$ $\operatorname{diag}(1,-1,-1,-1,-1)$ :

$$
\begin{aligned}
\mathcal{S}_{R}: & \eta_{A B} \xi^{A} \xi^{B}=-R^{2}, \\
& d s^{2}=\eta_{A B} d \xi^{A} d \xi^{B},
\end{aligned}
$$

where $R$ is the invariant length, and $A, B=0, \ldots, 4$. Clearly, Eqs. (2) and (3) are invariant under $d S$ group $\mathcal{G}_{R}=S O(1,4)$.

The Beltrami coordinates are defined patch by patch on $\mathcal{B}_{R} \simeq \mathcal{S}_{R}$. For intrinsic geometry of $\mathcal{B}_{R}$, there are at least eight patches $U_{ \pm \alpha}:=\left\{\xi \in \mathcal{S}_{R}: \xi^{\alpha} \gtrless 0\right\}, \alpha=1, \cdots, 4$. In $U_{ \pm 4}$, for instance, the Beltrami coordinates are

$$
\begin{aligned}
& \left.x^{i}\right|_{U_{ \pm 4}}=R \xi^{i} / \xi^{4}, \quad i=0, \cdots, 3 ; \\
& \left.\xi^{4}\right|_{U_{ \pm 4}}=\left(\xi^{0^{2}}-\sum_{a=1}^{3} \xi^{a 2}+R^{2}\right)^{1 / 2} \gtrless 0 .
\end{aligned}
$$

In the patches $\left\{U_{ \pm a}, a=1,2,3\right\}$,

$$
\left.y^{j^{\prime}}\right|_{U_{ \pm a}}=R \xi^{j^{\prime}} / \xi^{a}, \quad j^{\prime}=0, \cdots, \hat{a} \cdots, 4 ; \quad \xi^{a} \neq 0,
$$

where $\hat{a}$ means omission of $a$. It is important that all transition functions in intersections are of $\mathcal{G}_{R}$, say, in $U_{4} \cap U_{3}$, the transition function $T_{4,3}=\xi^{3} / \xi^{4}=x^{3} / R=$ $R / y^{4} \in \mathcal{G}_{R}$ so that $x^{i}=T_{4,3} y^{i^{\prime}}$ for $i=i^{\prime}=0,1,2$ and $x^{3}=R^{2} / y^{4}$. Note all of them are of type (11).

It is important that in each patch, there are condition and Beltrami metric

$$
\begin{aligned}
\sigma(x) & =\sigma(x, x):=1-R^{-2} \eta_{i j} x^{i} x^{j}>0, \\
d s^{2} & =\left[\eta_{i j} \sigma(x)^{-1}+R^{-2} \eta_{i k} \eta_{j l} x^{k} x^{l} \sigma(x)^{-2}\right] d x^{i} d x^{j} .(8)
\end{aligned}
$$

Under fractional linear transformations of type (1)

$$
\begin{aligned}
& x^{i} \rightarrow \tilde{x}^{i}= \pm \sigma(a)^{1 / 2} \sigma(a, x)^{-1}\left(x^{j}-a^{j}\right) D_{j}^{i}, \\
& D_{j}^{i}=L_{j}^{i}+R^{-2} \eta_{j k} a^{k} a^{l}\left(\sigma(a)+\sigma(a)^{1 / 2}\right)^{-1} L_{l}^{i}, \\
& L:=\left(L_{j}^{i}\right)_{i, j=0, \cdots, 3} \in S O(1,3),
\end{aligned}
$$

where $\eta_{i j}=\operatorname{diag}(1,-1,-1,-1)$ in $U_{ \pm \alpha}$, Eqs. (7) and (8) are invariant. Here (2) are the transitive part to the origin of $\mathcal{G}_{R}$. The inertial frames and inertial motions transform among themselves, respectively.

Note that Eqs. (7)-(9) are defined on $\mathcal{B}_{R}$ patch by patch. $\sigma(x)=0$ is the conformal boundary of $\mathcal{B}_{R}, \partial \mathcal{B}_{R}$.

The generators of $\mathcal{G}_{\Lambda}$ in Beltrami coordinates are expressed as

$$
\begin{aligned}
& \mathbf{P}_{i}=\left(\delta_{i}^{j}-R^{-2} x_{i} x^{j}\right) \partial_{j}, \quad x_{i}:=\eta_{i j} x^{j}, \\
& \mathbf{L}_{i j}=x_{i} \mathbf{P}_{j}-x_{j} \mathbf{P}_{i}=x_{i} \partial_{j}-x_{j} \partial_{i} \in \operatorname{so}(1,3) .
\end{aligned}
$$

They form an $s o(1,4)$ algebra

$$
\begin{aligned}
{\left[\mathbf{P}_{i}, \mathbf{P}_{j}\right] } & =R^{-2} \mathbf{L}_{i j} \\
{\left[\mathbf{L}_{i j}, \mathbf{P}_{k}\right] } & =\eta_{j k} \mathbf{P}_{i}-\eta_{i k} \mathbf{P}_{j} \\
{\left[\mathbf{L}_{i j}, \mathbf{L}_{k l}\right] } & =\eta_{j k} \mathbf{L}_{i l}-\eta_{j l} \mathbf{L}_{i k}+\eta_{i l} \mathbf{L}_{j k}-\eta_{i k} \mathbf{L}_{j l} .
\end{aligned}
$$


For two separate events $A\left(a^{i}\right)$ and $X\left(x^{i}\right)$ in $\mathcal{B}_{R}$,

$$
\Delta_{R}^{2}(A, X)=R\left[\sigma^{-1}(a) \sigma^{-1}(x) \sigma^{2}(a, x)-1\right]
$$

is invariant under transformations (9) of $\mathcal{G}_{R}$. Thus, the interval between $A$ and $B$ is timelike, null, or spacelike, respectively, according to

$$
\Delta_{R}^{2}(A, B) \gtreqless 0 .
$$

The proper length of timelike or spacelike between $A$ and $B$ are integral of $\mathcal{I} d s$ over the geodesic segment $\overline{A B}$ :

$$
\begin{aligned}
& S_{t-\text { like }}(A, B)=R \sinh ^{-1}(|\Delta(a, b)| / R), \\
& S_{s-\text { like }}(A, B)=R \arcsin (|\Delta(a, b)| / R),
\end{aligned}
$$

where $\mathcal{I}=1,-i$ for timelike or spacelike, respectively.

It can be shown that the light-cone at $A$ with running points $X$ is

$$
\mathcal{F}_{R}:=R\left\{\sigma(a, x) \mp[\sigma(a) \sigma(x)]^{1 / 2}\right\}=0 .
$$

It satisfies the null-hypersurface condition

$$
\left.g^{i j} \frac{\partial f}{\partial x^{i}} \frac{\partial f}{\partial x^{j}}\right|_{f=0}=0
$$

where $g^{i j}=\sigma(x)\left(\eta^{i j}-R^{-2} x^{i} x^{j}\right)$ is the inverse Beltrami metric. And at the origin of the Beltrami coordinates $a^{i}=0$, the light cone becomes a Minkowskian one and the invariant speed $c$ is numerically the velocity of light in the vacuum.

\section{INERTIAL MOTION, OBSERVABLE AND BELTRAMI SIMULTANEITY}

According to the PoR, a free particle with mass $m_{\Lambda 0}$ should move inertially along a timelike geodesic of the $B d S$ spacetime. It is just the case. The geodesic equation has the first integration

$$
\frac{d p^{i}}{d s}=0, \quad p^{i}:=m_{\Lambda 0} \sigma(x)^{-1} \frac{d x^{i}}{d s}=C^{i}=\text { const. }
$$

This implies under the initial condition

$$
x^{i}(s=0)=b^{i}, \quad \frac{d x^{i}}{d s}(s=0)=c^{i}
$$

with the constraint $g_{i j}(b) c^{i} c^{j}=1$, a new parameter $w=w(s)$ can be chosen such that the geodesic is just a straight world-line

$$
x^{i}(w)=c^{i} w+b^{i} .
$$

The parameter $w$ can be integrated out,

$$
w(s)= \begin{cases}R e^{\mp s / R} \sinh \frac{s}{R}, & \eta_{i j} c^{i} c^{j}=0, \\ \frac{R \sinh \frac{s}{R}}{\frac{\eta_{i j} c^{i} b^{j}}{R \sigma(b)} \sinh \frac{s}{R}+\cosh \frac{s}{R}}, & \eta_{i j} c^{i} c^{j} \neq 0 .\end{cases}
$$

Similarly, a light signal moves inertially along a null geodesic, which still has the first integration

$$
\sigma^{-1}(x) \frac{d x^{i}}{d \tau}=\text { constant }
$$

where $\tau$ is an affine parameter. Again, under the initial condition

$$
x^{i}(\tau=0)=b^{i}, \quad \frac{d x^{i}}{d \tau}(\tau=0)=c^{i} .
$$

and the constraint $g_{i j}(b) c^{i} c^{j}=0$, the null geodesic can be expressed as a straight line

$$
x^{i}=c^{i} w(\tau)+b^{i},
$$

where

$$
w(\tau)= \begin{cases}\tau, & \eta_{i j} c^{i} c^{j}=0, \\ -\frac{R^{2} \sigma(b)}{\left|\eta_{i j} c^{i} c^{j}\right|}\left(\frac{1}{\tau+\tau_{0}}-\frac{1}{\tau_{0}}\right), & \eta_{i j} c^{i} c^{j} \neq 0,\end{cases}
$$

with

$$
\tau_{0}=\sqrt{\frac{R^{2} \sigma(b)}{\left|\eta_{i j} c^{i} c^{j}\right|}}
$$

Thus, the motions of both free particles and light signals are indeed inertial, i.e. the coordinate velocity components are constants, respectively:

$$
\frac{d x^{a}}{d t}=v^{a} ; \quad \frac{d^{2} x^{a}}{d t^{2}}=0 ; \quad a=1,2,3 .
$$

Note that these properties are well defined patch by patch on whole $\mathcal{B}_{R}$.

Now we are ready to define the observables for free particles. From Eq. (18), it is natural to define the conservative quantities $p^{i}$ as the 4 -momentum of a free particle with mass $m_{\Lambda, 0}$ and its zeroth component as the energy. Note that it is no longer a 4 -vector rather a pseudo 4vector.

Furthermore, for a free particle a set of conserved quantities $L^{i j}$ may also be defined by

$$
L^{i j}=x^{i} p^{j}-x^{j} p^{i} ; \quad \frac{d L^{i j}}{d s}=0 .
$$

These may be called the 4-angular-momentum and they are also no longer an anti-symmetric tensor but a pseudo anti-symmetric tensor. However, $p^{i}$ and $L^{i j}$ constitute a conserved 5-d angular momentum for a free particle such that $\mathcal{L}^{i j}=L^{i j}, \mathcal{L}^{4 i}=p^{i}$. Then, we have

$$
\mathcal{L}^{A B}=m_{\Lambda 0}\left(\xi^{A} \frac{d \xi^{B}}{d s}-\xi^{B} \frac{d \xi^{A}}{d s}\right) ; \quad \frac{d \mathcal{L}^{A B}}{d s}=0 .
$$

For such a kind of free particles, there is a generalized Einstein's famous formula in $\mathcal{B}_{R}$ :

$$
-\frac{1}{2 R^{2}} \mathcal{L}^{A B} \mathcal{L}_{A B}=E^{2}-\boldsymbol{P}^{2}-\frac{1}{2 R^{2}} L^{i j} L_{i j}=m_{\Lambda 0}^{2},
$$


where $\mathcal{L}_{A B}=\eta_{A C} \eta_{B D} \mathcal{L}^{C D}, L_{i j}=\eta_{i k} \eta_{j l} L^{k l}$, italic blod $\boldsymbol{P}$ denotes the triple $\left(P^{1}, P^{2}, P^{3}\right), m_{\Lambda 0}$ introduced above should be the inertial mass for a free particle. It is well defined together with the energy, momentum and angular momentum at classical level.

It can further be shown that $m_{10}^{2}$ is the eigenvalue of the first Casimir operator of $\mathcal{G}_{R}[\underline{6}]$

$$
\mathbf{C}_{1}=\mathbf{P}_{i} \mathbf{P}^{i}-\frac{1}{2} R^{-2} \mathbf{L}_{i j} \mathbf{L}^{i j}
$$

with $\mathbf{P}^{i}=\eta^{i j} \mathbf{P}_{j}, \mathbf{L}^{i j}=\eta^{i k} \eta^{j l} \mathbf{L}_{k l}$. In addition, spin can also be defined through the eigenvalue of the second Casimir orperator

$$
\mathbf{C}_{2}=\mathbf{S}_{i} \mathbf{S}^{i}-R^{-2} \mathbf{W}^{2}
$$

as it was done in the relativistic quantum mechanics in Minkowski spacetime, where

$$
\begin{aligned}
& \mathbf{S}_{i}=\frac{1}{2} \epsilon_{i j k l} \mathbf{P}^{j} \mathbf{L}^{k l}, \quad \mathbf{S}^{i}=\eta^{i j} \mathbf{S}_{j}, \\
& \mathbf{W}=\frac{1}{8} \epsilon_{i j k l} \mathbf{L}^{i j} \mathbf{L}^{k l} .
\end{aligned}
$$

In Eq.(31), $\epsilon_{\mu \nu \lambda \sigma}$ is 4-d Levi-Civita symbol in flat spacetime with $\epsilon_{0123}=1$.

Thus, $\mathcal{S R}_{c, R}$ offers a consistent way to define the observable for free particles. Of course, these issues significantly confirm that the motion of a free particle in $\mathcal{B}_{R}$ is of inertia, the coordinate systems with Beltrami metric are inertial systems and corresponding observer at the origin of the system is of inertia as well.

Note that in order to make sense of inertial motions, etc., one should define simultaneity and take space-time measurements. In $\mathcal{S} \mathcal{R}_{c}$, coordinates have measurement significance that is linked with the PoR. Namely, the difference in time coordinate stands for the time interval, and the difference in spatial coordinate stands for the spatial distance. Similar to $\mathcal{S R}_{c}$, one can define that two events $A$ and $B$ are simultaneous if and only if the Beltrami time coordinate $x^{0}$ for the two events are same,

$$
a^{0}:=x^{0}(A)=x^{0}(B)=: b^{0} .
$$

It is called the Beltrami simultaneity and with respect to it that free particles move inertially. The Beltrami simultaneity defines a $3+1$ decomposition of spacetime

$$
d s^{2}=N^{2}\left(d x^{0}\right)^{2}-h_{a b}\left(d x^{a}+N^{a} d x^{0}\right)\left(d x^{b}+N^{b} d x^{0}\right)(33)
$$

with the lapse function, shift vector, and induced 3geometry on 3-hypersurface $\Sigma_{c}$ in one coordinate patch.

$$
\begin{aligned}
& N=\left\{\sigma_{\Sigma_{c}}(x)\left[1-\left(x^{0} / R\right)^{2}\right]\right\}^{-1 / 2}, \\
& N^{a}=x^{0} x^{a}\left[R^{2}-\left(x^{0}\right)^{2}\right]^{-1}, \\
& h_{a b}=\delta_{a b} \sigma_{\Sigma_{c}}^{-1}(x)-\left[R \sigma_{\Sigma_{c}}(x)\right]^{-2} \delta_{a c} \delta_{b d} x^{c} x^{d},
\end{aligned}
$$

respectively, where $\sigma_{\Sigma_{c}}(x)=1-\left(x^{0} / R\right)^{2}+\delta_{a b} x^{a} x^{b} / R^{2}$, $\delta_{a b}$ is the Kronecker $\delta$-symbol, $a, b=1,2,3$. In particular, at $x^{0}=0, \sigma_{\Sigma_{c}}(x)=1+\delta_{a b} x^{a} x^{b} / R^{2}$. In a vicinity of the origin of Beltrami coordinate system in one patch, 3 -hypersurface $\Sigma_{c}$ acts as a Cauchy surface.
The Beltrami simultaneity defines the laboratory time in one patch. According to the spirit of $\mathcal{S R}_{c}$ as well as the PoR, the Beltrami coordinates define, in such a manner, the standard clocks and standard rulers in laboratory of $\mathcal{S R}_{c, R}$ on $\mathcal{B}_{R}$. To measure the time of a process or the size of an object, we just need to compare with Beltrami coordinates.

\section{PROPER-TIME SIMULTANEITY AND ITS COSMOLOGICAL SIGNIFICANCE}

There is another simultaneity, however, in $\mathcal{S R}_{c, R}$. It is proper-time simultaneity with respect to a clock rest at spatial origin of the Beltrami coordinate system. Such a proper time $\tau_{\Lambda>0}$ of a rest clock on the time axis of Beltrami coordinate system, $\left\{x^{a}=0\right\}$, reads

$$
\tau_{\Lambda>0}=R \sinh ^{-1}\left(R^{-1} \sigma^{-\frac{1}{2}}(x) x^{0}\right) .
$$

Therefore, we can define that the events are simultaneous with respect to it if and only if

$$
x^{0} \sigma^{-1 / 2}(x, x)=\xi^{0}:=R \sinh \left(R^{-1} \tau\right)=\text { constant. }
$$

The line-element on the simultaneous 3-d hypersurface, denoted by $\Sigma_{\tau}$, can be defined as

$$
d l^{2}=-d s_{\Sigma_{\tau}}^{2}
$$

where

$$
\begin{aligned}
& d s_{\Sigma_{\tau}}^{2}=R_{\Sigma_{\tau}}^{2} d l_{\Sigma_{\tau} 0}^{2}, \\
& R_{\Sigma_{\tau}}^{2}:=\sigma^{-1}(x, x) \sigma_{\Sigma_{\tau}}(x, x)=1+\left(\xi^{0} / R\right)^{2} \\
& \sigma_{\Sigma_{\tau}}(x, x):=1+R^{-2} \delta_{a b} x^{a} x^{b}>0 \\
& d l_{\Sigma_{\tau} 0}^{2}:=\left\{\delta_{a b} \sigma_{\Sigma_{\tau}}^{-1}(x)-\left[R \sigma_{\Sigma_{\tau}}(x)\right]^{-2} \delta_{a c} \delta_{b d} x^{c} x^{d}\right\} d x^{a} d x^{b}
\end{aligned}
$$

It should be pointed out that this simultaneity is closely linked with the cosmological principle. In fact, it is significant that if $\tau_{\Lambda>0}$ is taken as a "cosmic time", the Beltrami metric (8) becomes an RW-like metric with a positive spatial curvature and the simultaneity is globally defined in whole $\mathcal{B}_{R}$

$$
d s^{2}=d \tau^{2}-d l^{2}=d \tau^{2}-\cosh ^{2}\left(R^{-1} \tau\right) d l_{\Sigma_{\tau} 0}^{2} .
$$

This shows that the 3-d cosmic space is $S^{3}$ rather than flat. The deviation from the flatness is of order $\Lambda$. Obviously, this spatial closeness of the universe is a remarkable property different from the standard cosmological model with flatness. This property seems more or less already indicated by the CMB power spectrum from WMAP 1], 2] and should be further checked by its data in large scale.

The two definitions of simultaneity do make sense in different kinds of measurements. The first concerns the measurements in a laboratory and is related to the PoR of $\mathcal{S} \mathcal{R}_{c, R}$. The second concerns with cosmological effects. Furthermore, the relation between the Beltrami metric and its RW-like counterpart (39) in terms of the coordinate time $x^{0}$ and the cosmic time $\tau$ links the PoR and cosmological principle. It is very meaningful. 


\section{REMARKS}

We have set up the special relativity with an invariant length $R$ in addition to $c, \mathcal{S R}_{c, R}$ of $\mathcal{B}_{R}$, with $d S$ invariance based on the PoR and PoI. Similarly, $\mathcal{S} \mathcal{R}_{c, R}$ with $A d S$ invariance can also be set up. The Beltrami coordinates are of inertia, the test particles and signals move inertially along the timelike, null straight world lines, respectively. Their classical observable can be well defined. And, the famous Einstein's energy-momentum-mass relation is generalized in $\mathcal{S R}_{c, R}$.

The relation between the Beltrami metric and the RWlike metric in $\mathcal{S} \mathcal{R}_{c, R}$ of $\mathcal{B}_{R}$ indicates a relation between the PoR and cosmological principle and predicts that the 3 - $\mathrm{d}$ cosmic space is slightly closed.

It should be noted that all properties in $\mathcal{S} \mathcal{R}_{c, R}$ of $\mathcal{B}_{R}$ are in analog with $\mathcal{S} \mathcal{R}_{c}$ and coincide with it if $R \rightarrow \infty$.

In fact, all possible kinematics with ten-parameter transformation groups [15] should be contracted [16] from the relativity with invariant speed and length $\mathcal{S R}_{c, R}$. For instance, one may consider the Newton-Hooke limit 17], 18], 19] of the $d S$ spacetime. However, as was mentioned in 5], the Newton-Hooke limit is Not unique. In order to fix the Newton-Hooke limit uniquely, one should require the Galilei-Hooke's relativity principle as well as the principle of infinite velocity of signal, which are corresponding to the $\mathrm{PoR}$ and the PoI here, respectively. Thus, the Newton-Hooke space-time given in [5] is the Newton-Hooke contraction of the relativity $\mathcal{S} \mathcal{R}_{c, R}$ on $\mathcal{B}_{R}$.
Finally, we should mention the relation between the relativity with invariant speed and length, $\mathcal{S} \mathcal{R}_{c, R}$, presented here and the DSR [13]. In fact, $\mathcal{S R}_{c, R}$ is a relativity with the both observer-independent invariants, velocity scale and length scale, in configuration space, while the DSR is the one involves an observer-independent largevelocity scale and an observer-independent small-length scale/large-momentum scale. It has been noticed, however, that the DSR may be viewed as a theory with energy-momentum space being the 4 -d $d S$ space and that the inhomogeneous projective coordinates are employed [20], which are locally the same as the Beltrami coordinates except the latter without the antipodal identification. This may be traced back to 21. However, since the antipodal identification had been used there, resulting in the energy-momentum space neither orientable nor energy orientable. Thus, we may directly regard $\mathcal{S} \mathcal{R}_{c, R}$ as the counterpart of DSR in its momentum space without orientation problem as long as $R$ is taken as the observerindependent large-momentum scale.

\section{Acknowledgments}

The authors would like to thank Professors Z. Chang, G. Gibbons, Q. K. Lu, J. Z. Pan and Y.S. Wu as well as Drs. Y. Ling and Y. Tian for valuable discussions. This work is partly supported by NSFC under Grants Nos. 90103004, 10175070, 10375087.
[1] C. L. Bennett, et al, Astrophys.J. (Suppl.) 148 (2003) 1, arXiv: astro-ph/0302207

[2] M. Tegmark, et al, arXiv: astro-ph/0310723

[3] A. Strominger, Talk at the String Satellite Conference to ICM 2002. August, 2002, Beijing.

[4] H.Y. Guo, C.G. Huang, Z. Xu and B. Zhou, arXiv: hep-th/0311156

[5] H.Y. Guo, C.G. Huang, Y. Tian, Z. Xu and B. Zhou, arXiv: hep-th/0403013

[6] F. Gürsey, in Relativity, Groups and Topology, ed. C. DeWitt and B. DeWitt, (Blackie and Son Ltd,1969).

[7] K.H. Look (Qi-Keng Lu)(1970), "Why the Minkowski metric must be used?" unpublished.

[8] K.H. Look, C.L. Tsou (Z.L. Zou) and H.Y. Kuo (H.Y. Guo), Acta Physica Sinica 23 (1974) 225 (in Chinese); Nature (Shanghai, Suppl.), Modern Physics 1 (1980) 97 (in Chinese).

[9] L.G. Hua, (1974), An unpublished manuscript.

[10] Zhan Xu (1974-1975), Private communications.

[11] H.Y. Kuo, Proceedings of the 2nd Marcel Grossmann Meeting on General Relativity, ed. by R. Ruffini, (NorthHolland Pub. Amsterdam, 1982) 801-807; H.Y. Guo, Nucl. Phys. B (Proc. Suppl.) 6 (1989) 381.
[12] E. Beltrami, Opere Mat., 1 (1868) 374-405.

[13] G. Amelino-Camelia, Int. J. Mod. Phys. D11 (2002) 35; Nature 418 (2002) 34; Phys.Lett. B510 (2001) 255.

[14] V. Fock, The Theory of Space Time and Gravitation, Pergamon Press (1964).

[15] H. Bacry and J.M. Lévy-Leblond, J. Math. Phys. 9 (1968) 1605; H. Bacry and J. Nuyts, J.Math.Phys. 27 (1986) 2455; J.R. Derome and J.G. Dubois, IL Nuovo Cimento 9 (1972) 351.

[16] E. Inönü and E.P. Wigner, Proc. Nat. Acad. Sci. 39(1953) 5.

[17] R. Aldrovandi, A.L. Barbosa, L.C.B. Crispino and J.G. Pereira, Class.Quant.Grav. 16 (1999) 495-506, arXiv: gr-qc/9801100

[18] Yi-Hong Gao, arXiv: hep-th/0107067

[19] G.W. Gibbons, C.E. Patricot, Class.Quant.Grav. 20 (2003) 5225, arXiv: hep-th/0308200 and references therein.

[20] J. Kowalski-Glikman, Phys. Lett. B547 (2002) 291; J. Kowalski-Glikman and S. Nowak, Class.Quant.Grav. 20 (2003) 4799.

[21] H.S. Snyder, Phys. Rev. 71 (1947) 38. 